\title{
Advances in the simulation of autonomous microwave circuits
}

\author{
A. Suárez, S. Sancho, M. Pontón, F. Ramírez \\ Universidad de Cantabria, Santander, 39005, suareza@unican.es
}

\begin{abstract}
Advances in the simulation of nonlinear microwave circuits of autonomous nature are presented, covering three relevant aspects: potential instability of power amplifiers under output mismatch effects, design of dual-band frequency dividers, with interest in multi-band communication systems, and oscillator phase-noise analysis. The instability under mismatch effects is predicted through the calculation of a 3-port scattering matrix, at three relevant sideband frequencies, which is obtained by linearizing the circuit about the large-signal steady-state solution. The dual-band divider is based on a parallel configuration, using two types of inductor-varactor cells, as well as a simultaneous design/optimization of two identical circuits, each operating at one of the two frequency bands. Finally, a new phase-noise analysis method able to predict the near-carrier spectral density in the presence of near-critical poles is described.
\end{abstract}

Index Terms - Power amplifier, frequency divider, oscillator, stability, phase noise.

\section{INTRODUCTION}

Advances in critical aspects of the nonlinear simulation of circuits of autonomous nature, or capable to exhibit oscillations, are presented. This oscillation can be required for the circuit intended operation, as in the case of local oscillators and frequency dividers, or undesired, as in the case of a power amplifier (PA). The three aspects addressed here are the stability analysis of PAs under output mismatch effects, the design of dual-band frequency dividers and the accurate prediction of the oscillator phase noise.

PAs that are stable under a standard $50 \Omega$ termination may become unstable when connected to an antenna, due to reflection effects in realistic wireless environments [1]. The stability analysis must be carried out under unknown termination impedances and must cover all the possible combinations of the impedances at the relevant harmonic and sideband frequencies. This is done through the calculation of a scattering-type matrix, obtained by linearizing the circuit about the large-signal steady-state solution, depending on the mismatched impedance termination at the fundamental frequency $f_{\text {in }}$ [2]. The virtual ports of this matrix will correspond to the sideband frequencies with impact on the stability properties. The potential instability is predicted by plotting a global large-signal stability factor on the Smith Chart associated with the impedance termination at $f_{i n}$.

Recently, a frequency-divider topology based on nonlinear transmission lines NLTL has been proposed [3], having the advantages of zero static-power consumption, no free-running oscillation and a low division threshold. The combination of two types of L-varactor cells can enable a division in two distinct frequency bands [4]. A simulation method based on the simultaneous optimization/design of two circuit replicas, each operating at one of the two bands, allows presetting the division bands and lowering the input-amplitude threshold.
Oscillator phase noise is the third aspect considered. It is an undesired characteristic of local oscillators, which may lead to demodulation errors. Various time and frequency domain methods have been proposed in the literature [5-6]. However, some limitations still exist, such as the prediction of the near carrier noisy spectrum in the presence of flicker noise. This will be addressed with a new frequency-domain method, based on the calculation of the variance of the phase deviation [7] and able account for the effect of near-critical poles.

\section{MismatCH-INDUCED InSTABILITY OF PAS}

The stability analysis under mismatch effects is carried out at the PA output reference plane, where it is connected to the antenna [Fig. 1(a)]. The impedance terminations at the harmonic frequencies $k f_{i n}$, where $k$ is an integer, will have an impact on the large-signal periodic solution and those at the sideband frequencies will have an impact on the circuit linearization with the conversion-matrix approach [2]. In most cases, and due to the filtering effects of the output network, the only sensitive impedance terminations will correspond to $f_{\text {in }}$, $f_{b}=f, f_{l}^{*}=f_{\text {in }}+f$ and $f_{u}=f_{\text {in }}+f$, where $f$ is the perturbation frequency, which mixes with $f_{i n}$. A $3 \times 3$ impedance matrix $\left[Z_{3}\right]$ will be obtained considering 3 virtual ports at $f_{b}, f_{l}^{*}$ and $f_{u}$ [Fig. 1(b)]. The rest of frequencies, arbitrarily terminated in $50 \Omega$, are taken into account in an inner tier.

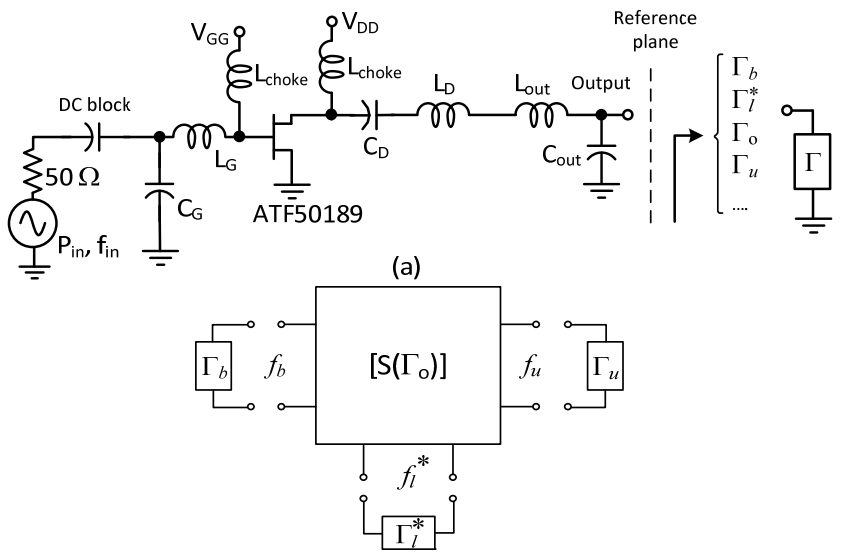

(b)

Fig. 1. PA under mismatch effects. (a) Schematic and reference plane. (b) Scattering matrix describing the mismatched PA response at 3 sidebands.

The matrix $\left[Z_{3}\right]$ is calculated by sequentially exciting the circuit with a small-signal current source at $f_{b}, f_{l}^{*}$ and $f_{u}$. In this calculation, performed in a certain $f$ interval, the circuit is linearized about the steady-state solution obtained with the load $\Gamma_{\mathrm{o}}$ at $f_{\text {in }}$. The matrix $\left[\mathrm{Z}_{3}\right]$ is transformed into scattering matrix 
[S $\mathrm{S}_{3}$, which can be reduced to a $2 \times 2$ one when considering a particular termination $\Gamma_{s b}$ at any of the three sidebands. This way it is possible to obtain 3 pairs of large-signal stability factors, which agree with the large-signal equivalents of the $\mu$ factors in [8]. It is easily shown [2] that the most critical stability situation corresponds to $\Gamma_{s b}=1 e^{j \phi}$. When sweeping $\phi$ from $0^{\circ}$ to $360^{\circ}$, and taking the minima of any of the large-signal $\mu$ factors, the same prediction on the circuit potential instability is obtained, as shown in Fig. 2 , where the factors $\mu_{b, u}$ , $\mu_{b, l}$ and $\mu_{l, u}$ have been represented versus $f$, and the subindexes refer to the selected ports. For absolute stability at $\Gamma_{o}$ and $f$, the factors must be larger than 1 . To determine the terminations $\Gamma_{o}$ giving rise to potential instability, one should trace the contour plot $\mu_{L S}^{\min }\left(\Gamma_{o}\right)=\min _{f}\left[\mu_{L S}\left(\Gamma_{o}, f\right)\right]=1$, where $\mu_{L S}$ refers to any of the large-signal factors. This analysis has been carried out in Fig. 3(a) and has been validated experimentally using a triple-stub tuner [Fig. 3(b)]. The ensemble of spectra corresponding to the stable and unstable loads is shown in Fig. 3(c) and 3(d), respectively.

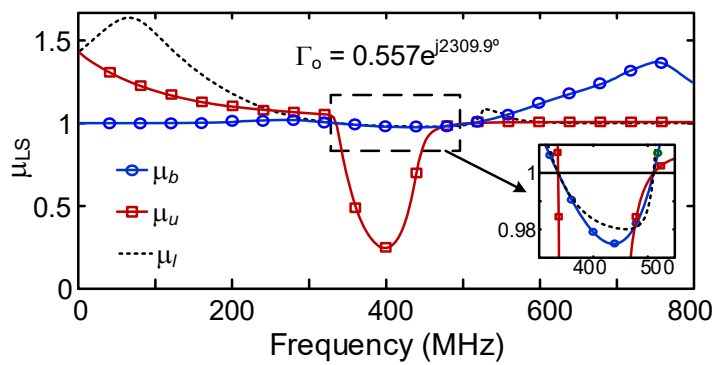

Fig. 2 Variation versus the perturbation frequency $f$ of the minima of the 3 factors $\mu_{b, u}(\phi), \mu_{b, l}(\phi)$ and $\mu_{l, u}(\phi)$ with $\left|\Gamma_{s b}\right|=1$, for $\Gamma_{\mathrm{o}}=0.577 \mathrm{e}^{\mathrm{j} 150^{\circ}}$.

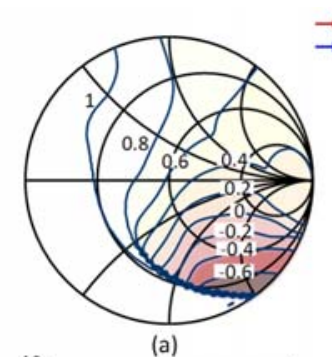

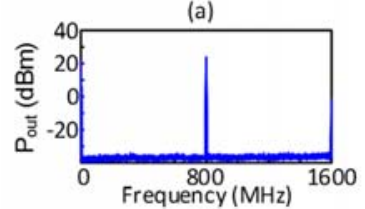

(c)

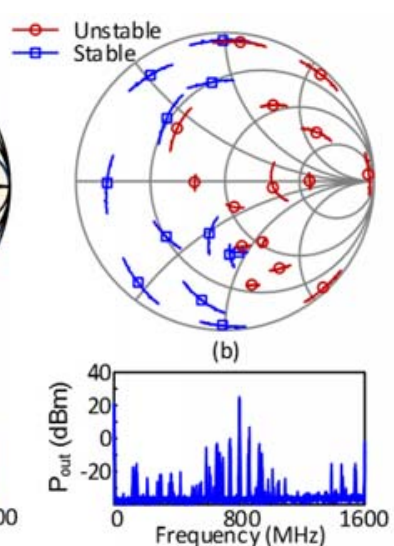

(d)
Fig. 3 (a) Contour plot of $\mu_{L S}^{\min }\left(\Gamma_{o}\right)$. (b) Exhaustive experimental validation with a triple stub tuner. (b) Ensemble of output spectra obtained for plots marked with squares (stable behavior). (c) Ensemble for plots marked with circles (unstable behavior).

\section{DUAL-BAND FREQUENCY DIVIDER}

The dual-band frequency divider by 2 is based on the composition of three L-varactor cells, two of them having the varactors in parallel, and the middle one having the varactors in series (Fig. 4). Element values in the series-varactor (parallelvaractor) cell are chosen to have a small impact on the higher (lower) frequency band. Thus an initial independent design of the cells providing each division band $(1.72 \mathrm{GHz}$ and 3.97 $\mathrm{GHz}$ ) can be carried out [4]. The band is centred by tuning the inductors $L_{l}$ and $L_{u}$, and the capacitors $c_{l}$ and $c_{u}$ are used to reduce the division threshold. Due to coupling effects, one cannot center one band without affecting the other, as gathered from the sensitivity curves of Fig. 5(a) and (b). This is avoided through the simultaneous optimization of two circuit copies, with identical element values, and using the results of the separate designs as an initial guess. Each copy operates at one of the bands, with an auxiliary generator (AG) at the $f_{\text {in }} / 2$ [4]. Two-tone HB is used with 0 intermodulation order. To preset the division threshold, the AG amplitudes are set to a very small value $\varepsilon$. The optimization conditions are:

$$
\begin{aligned}
& Y_{A G l}=\left(L_{l}, c_{l}, L_{u}, c_{u}, \phi_{i n, l}\right)=0 \\
& Y_{A G u}=\left(L_{l}, c_{l}, L_{u}, c_{u}, \phi_{i n, u}\right)=0
\end{aligned}
$$

where $\phi_{i n, l}, \phi_{i n, u}$ are the respective input-source phases. The independent simulation of Fig. 5(c) confirms the division-band centring. Measurements are superimposed.
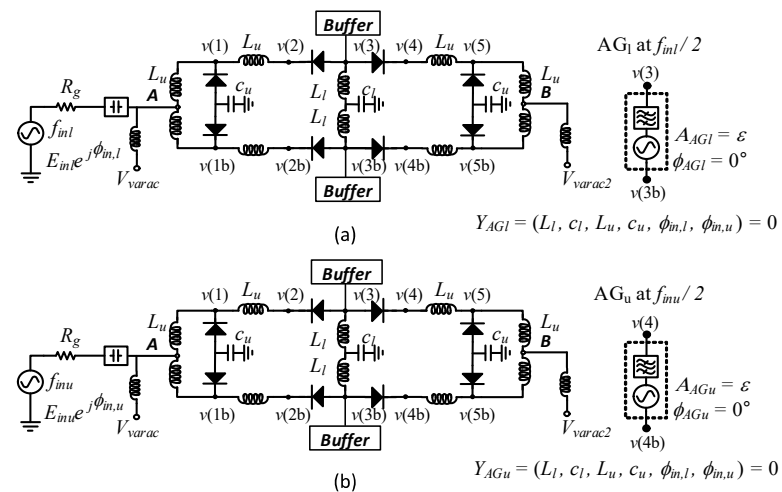

Fig. 4 Dual-band frequency divider. The circuit is duplicated for the simultaneous pre-setting of the two division bands, using two AGs at the frequencies $f_{A G l}=f_{i n l} / 2$ and $f_{A G u}=f_{i n u} / 2$.

\section{Phase Noise ANALYsis In Oscillator Circuits}

In the presence of noise sources, the amplitude perturbation of a stable oscillator remains small, whereas the phase perturbation is unbounded. Thus, the power spectral density (PSD) of the noisy state variables about each $k$-th harmonic component is mainly due to the phase perturbation [5]:

$$
\begin{aligned}
& S_{i, k}(f) \cong F\left\{R_{i, k}(\tau)\right\}, R_{i, k}(\tau)=\exp \left\{-\frac{\sigma_{i, k}^{2}(|\tau|)}{2}\right\} \\
& \sigma_{i, k}^{2}(t, \tau)=\left\langle\left[\Delta \phi_{i, k}(t+\tau)-\Delta \phi_{i, k}(t)\right]^{2}\right\rangle \cong \sigma_{i, k}^{2}(|\tau|)
\end{aligned}
$$

where $F$ is the Fourier transform operator and $f$ is the frequency offset from the carrier at $k f_{o}$, with $f_{o}$ being the fundamental frequency. The process $\Delta \phi_{i, k}(t)$ represents the phase 
perturbation component corresponding to the $k$-th harmonic of the $i$-th state variable of the oscillator. It can be demonstrated [7] that, even in the presence of flicker noise sources, in most practical measurement procedures the variance $\sigma_{i, k}^{2}(|\tau|)$ can be associated to a nearly-stationary process, so the dependence on $t$ can be neglected.

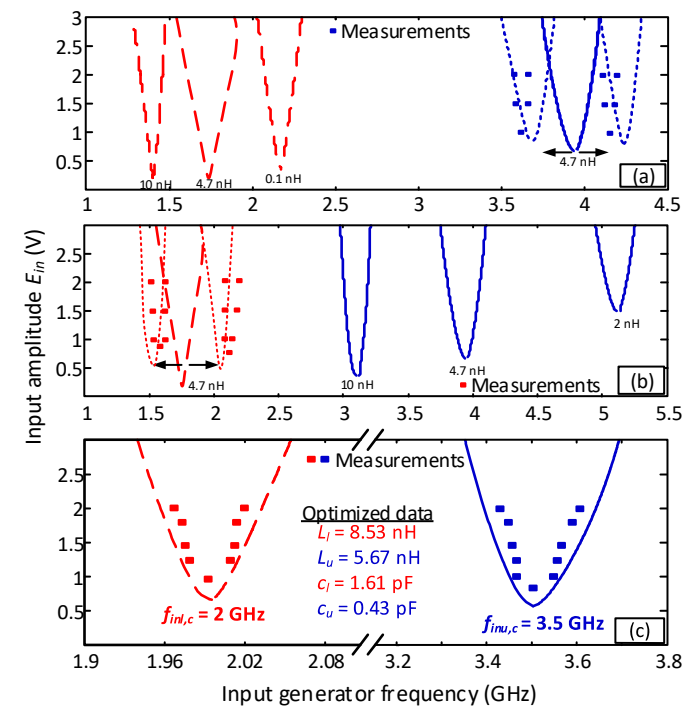

Fig. 5 Frequency-divider sensitivity curves, with measurements superimposed. (a) and (b) Independent designs (c) Simultaneous optimization.

The analysis method is based on the calculation of the phase perturbation by means of a semi-analytical formulation of the oscillator [9], in which the circuit equations are compacted into a first-harmonic admittance equation at one observation node $q$, given by $Y(V, \omega) X_{1}=0$, which acts like an outer tier. The phase and amplitude of the first harmonic $X_{1}$ of the voltage $v_{q}(t)$ will be perturbed by the noise sources. There are two different ways to represent these perturbations, leading to the following expressions:

$$
\begin{aligned}
& \text { a) } X_{1}(t)=\left[V_{1}+\Delta V_{1}(t)\right] \exp \left\{j\left[\phi_{1}+\Delta \phi(t)\right]\right\} \\
& \text { b) } X_{1}(t)=\left[X_{1}^{0}+\Delta X(t)\right] \exp \{j k \Delta \phi(t)\}, \Delta X \in C
\end{aligned}
$$

Expressions $(3)(\mathrm{a}, \mathrm{b})$ correspond to a Conversion Matrix type approach [10] and a Carrier Modulation type approach [6], respectively. Now, introducing expression (3)(a) or (3)(b) in the semi-analytical admittance equation, one obtains the following Laplace-domain equation for the first-harmonic phase perturbation [4]:

$$
\Delta \phi(s)=\frac{1}{s}\left[\bar{c}_{w}^{+}(s) \bar{I}_{w}(s)+c_{c}(s) I_{c}(s)\right]
$$

where $\bar{I}_{w}, I_{c}$ are the current sources, entering the node $q$, that model the white and colored noise [5]. The transfer functions in $\bar{c}_{w}(s), c_{c}(s)$ depend on the decomposition [(3)(a) or (3)(b)] used for the calculation. When using (3)(a), $\bar{c}_{w}(s)$ and $c_{c}(s)$ contain the poles resulting from the stability analysis of the oscillatory solution. If these poles, which are transferred to $S_{i, 1}(f)$ through (2), are close to the imaginary axis, they will have an impact on the noise spectrum. When using (3)(b), the functions $\bar{c}_{w}(s), c_{c}(s)$ become constant values, not affected by the dominant poles. Fig. 6 presents the PSD $S_{i, 1}(f)$ of a BJTbased oscillator at $1 \mathrm{GHz}$ [11]. The analysis based on (2) is able to predict the spectrum flattening near the carrier. There exists a pole with small real part $\lambda \cong-10^{6} s^{-1}$, giving rise to a change in the slope at about $30 \mathrm{KHz}$, only detected when using the decomposition (3)(a).

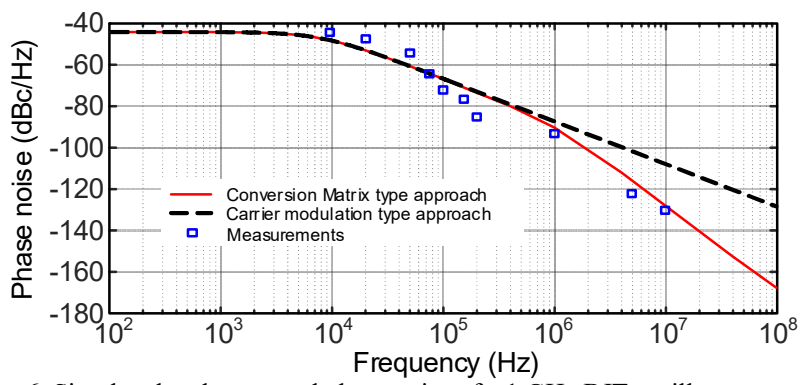

Fig. 6. Simulated and measured phase noise of a $1 \mathrm{GHz}$ BJT oscillator.

\section{ACKNOWLEDGMENT}

Work funded by the Spanish Ministry of Economy and Competitiveness (TEC2014-60283-C3-1-R), ERDF/FEDER and the Parliament of Cantabria (12.JP02.64069).

\section{REFERENCES}

[1] J. F. Imbornone, M. Murphy, R .S. Donahue and E. Heaney, "New insight into subharmonic oscillation mode of GaAs power amplifiers Under Severe Output Mismatch Condition," IEEE J. Solid-State Circuits, vol. 32, pp. 1319-1325, Sept., 1997.

[2] A. Suárez, F. Ramírez, S. Sancho, "Generalized stability criteria for power amplifiers under mismatch effects," IEEE Trans. Microw. Theory Techn., vol. 63, no. 12, pp. 4415-4428, 2015.

[3] W. Lee and E. Afshari, "Distributed Parametric Resonator: A Passive CMOS Frequency Divider," IEEE J. Solid-State Circuits, vol.45, no.9, pp.1834-1844, Sept., 2010.

[4] M. Pontón, A. Suárez, "Optimized Design of Frequency Dividers Based on Varactor-Inductor Cells," IEEE Trans. Microw. Theory Techn., vol. 63, no. 12, pp. 4458-4472, 2015.

[5] F. X. Kaertner, "Analysis of white and f-alpha noise in oscillators," Int. J. Circ. Theor. Appl., vol. 18, pp. 485-519, 1990.

[6] V. Rizzoli, F. Mastri, D. Masotti, "General noise analysis of nonlinear microwave circuits by the piecewise harmonic balance technique," IEEE Trans. Microw. Theory Tech., vol. 42, pp. 807$819,1994$.

[7] S. Sancho, A. Suarez, J. Dominguez and F. Ramirez, "Analysis of Near-Carrier Phase-Noise Spectrum in Free-Running Oscillators in the Presence of White and Colored Noise Sources," IEEE Trans. Microw. Theory Tech., vol. 58, pp. 587-601, 2010.

[8] D. Woods, "Reappraisal of the unconditional stability criteria for active 2-port networks in terms of S parameters," IEEE Trans. Circuits Syst., vol.23, no. 2, pp. 73-81, Feb., 1976.

[9] F. Ramirez, M. Ponton, S. Sancho and A. Suarez, "Phase noise of injection-locked oscillators and frequency dividers," IEEE Trans. Microw. Theory Tech., vol. 56, pp. 393-407, Feb., 2008

[10] J. M. Paillot, et al, "General program for steady state, stability, and FM noise analysis of microwave oscillators," IEEE MTT-S Int. Microw. Symp., vol. 3, pp. 1287-1290, 1990.

[11] S. Sancho, A. Suarez and F. Ramirez, "General Phase-Noise Analysis From the Variance of the Phase Deviation," IEEE Trans. Microw. Theory Tech., vol. 61, pp. 472-481, 2013. 